\title{
Mean-square exponential input-to-state stability of stochastic recurrent neural networks with multi-proportional delays *
}

\author{
Liqun Zhou*, Xueting Liu \\ School of Mathematics Science, Tianjin Normal University, Tianjin, 300387, \\ China
}

\begin{abstract}
This paper investigates mean-square exponential input-to-state stability of stochastic recurrent neural networks with multi-proportional delays. Here, we study the proportional delay, which is a kind of unbounded time-varying delay in stochastic recurrent neural networks, by employing Lyapunov-Krasovskii functional, stochastic analysis theory and Itô's formula. A new stability criterion about the mean-square exponential input-to-state stability, which is different from the traditional stability criteria, is presented. In addition, the new proposed criterion easy to verify and less conservation than earlier publications about mean-square exponential inputto-state stability of stochastic recurrent neural networks. Finally, several examples and their simulations are given to illustrate the correctness and effectiveness of the theoretical results.
\end{abstract}

Key words: Stochastic neural networks; Proportional delay; Mean-square exponential input-to-state stability; Lyapunov-Krasovskii functional

\section{Introduction}

As a class of fixed weights, external input and internal state neural networks, recurrent neural networks were diffusely known. Recurrent neural networks mainly contain Hopfield neural networks, cellular neural networks and CohenGrossberg neural networks. In view of their characteristics with fixed weights,

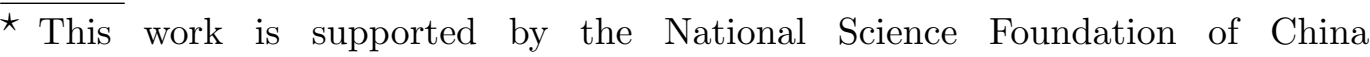
(No.61374009)

* Corresponding author.

Email address: zhouliqun20000@163.com
} 
recurrent neural networks were facilitated to hardware circuit implementations. Recurrent neural networks were widely discussed due to their potential applications in a diverse range of areas, such as phase structure, patterns recognition, associative memory, electronic olfactory system, optimization, wireless communications and so on[1-3]. The dynamic behaviors of the neural network can be subject to a variety of external disturbances, such as control input, noise disturbances, impulsive disturbances, random variable and so on. Furthermore, with the rapid development of science and technology, real systems become more and more complex. Actually, they are constantly influenced by many external disturbances which in many cases are regarded as random. Just as pointed out by Haykin [4], the synaptic transmission in real neural networks can be viewed as a noisy process introduced by random fluctuations from the release of neurotransmitters and other probabilistic causes. For this reason, the study on the dynamic behavior of stochastic neural networks have become a hot direction. As we all know, it is an extremely important object to study the stability issue of the stochastic neural networks since it is the presupposition that a real system can run normally and successfully. Fatally, in real system time delay is often appeared owing to the finite switching speed of amplifiers or information processing, and it is one of the most important factors that cause the system oscillation, chaos and unstable. So there is an important guiding significance to investigate the problem of stochastic delayed neural networks.

Thankfully, there are abundant stability results about the stochastic neural networks with different kinds of delays, for instance, see [5-19] and references therein. For example, in [5], Li Dan and Zhu Quanxin introduced and proposed a new comparison principle to study the stochastic delayed neural networks. By applying Lyapunov functional, stochastic analysis technique and Hardy inequality, respectively, exponential stability and global stochastic stability of the stochastic delayed neural networks with Markovian switching were investigated [6,7]. In [8,9] the stability of Markovin jumping stochastic CohenGrossberg neural networks was investigated. On basis of linear matrix inequality, a delay-dependent mean square exponential stability criterion is derived for the stochastic switched static neural networks via a Lyapunov-Krasovskii functional in $[10,11]$. Pth moment exponential stability was discussed in [12] by using fixed point theory for a class of stochastic delayed recurrent neural networks with impulses. Based on Razumikhin technique, Peng and Huang in [15] further studied exponential stability of hybrid stochastic recurrent neural networks with time-varying delays, and [16] studied a class of impulsive discrete-time stochastic BAM neural networks with time-varying delay were investigated by using difference inequalities and Lyapunov functional together with Razumikhin technique. In [17] Meng etc. further discussed the both Pth moment exponential stability and almost sure exponential stability of a class of stochastic recurrent neural networks with unbounded time-varying delays via Lyapunov functional and the semi-martingale convergence theorem. Glob- 
al exponential synchronization for coupled switched delayed recurrent neural networks with stochastic perturbation and impulsive effects were deeply investigated in [18]. In [19], the global robust stability problem of TS fuzzy Hopfield neural networks with parameter uncertainties and stochastic perturbations was investigated based on the Lyapunov method and stochastic analysis approaches, the delay-dependent stability criterion was derived in terms of linear matrix inequalities (LMIs).

It is worth putting forward that all of the above-mentioned works on the stability of stochastic delayed neural works were exponential stability, global asymptotical stability, almost surely stability and so on. As far as we know, the traditional stability means that the states of stochastic neural networks converge to the equilibrium point as time goes to infinity. But this is not always what happens in our real lives. For examples, some systems such as the pendulum and stock markets only remain bounded but do not necessarily converge to the equilibrium point. Actually, this phenomenon frequently arises in lots of realistic systems, such as network control systems. Hence, it is important to investigated the input-to-state stability problem in the area of stochastic neural networks. Until now, there are some results about the input-to-state stability of stochastic delayed neural networks. For instance, see $[20,22,23]$ and references therein. On the basis of Lyapunov functional, two algebraic criteria for input-to-state stability of recurrent neural networks with time-varying delays were obtained [20].Yang in [22] studied exponential input-to-state stability of recurrent neural networks with multiple timevarying delays by utilizing Lyapunov-Krasovskii functional and linear matrix inequalities techniques. Mean-square exponential input-to-state stability was addressed in [23] for a class of stochastic delayed neural networks by constructing Lyapunov-Krasovskii functional,using stochastic analysis theory and Itô's formulas.

Proportional delay is a special type among delays, which is time-varying and unbounded, which is different from constant delay, bounded time-varying delay $[13,14,21,24]$ and distributed delay, etc. The proportional delay function $\tau(t)=(1-q) t \rightarrow+\infty$ as, $q \neq 1, t \rightarrow+\infty$, where $q$ is a constant, and satisfies $0<q \leq 1$. Just as pointed out by Zhou [29], although proportional delay and distributed delay are all unbounded time-varying delay, there is a big difference between the them, proportional delay is not easy to deal with in the derivation of dynamic behavior of neural networks. Proportional delay is an objective existence, such as in Web quality of service (QoS) routing decision, proportional delay is usually required. Furthermore, in many fields such as IP networks and signal processing, proportional delays always play an important role during the design of a system's model [25-28]. A neural network usually has a spatial nature due to the presence of an amount of parallel pathways of a variety of axon sizes and lengths, so based on the network topology and material, it is possible to introduce the proportional delays in neural networks in some 
cases. Up to now, there are several achievements of dynamical behaviors for neural networks with proportional delays [29-36]. Based on the inner product properties, dissipativity of a class of cellular neural networks with proportional delays were discussed in [29]. In [30-32] the global exponential stability of cellular neural networks with multi-proportional delays were investigated by applying matrix theory and constructing Lyapunov functional, respectively. Song and Peng in [33] studied global asymptotic stability of impulsive cellular neural networks with proportional delays and partially Lipschitz activation functions via relative nonlinear measure. In [34], novel global exponential stability criteria for hybrid BAM neural networks with proportional delays is presented by constructing appropriate delay differential inequalities. In [35], by constructing Lyapunov functional and establishing the delay differential inequality, several delay-dependent and delay-independent sufficient conditions are derived to ensure global exponential periodicity and stability of recurrent neural networks with multi-proportional delays. In [36], the stability of impulsive recurrent neural networks with proportional delays is investigated by using the fixed point theory and some analysis techniques. The advantage of neural networks with proportional delays is that the network's running time can be controlled according to the network allowed delays. Besides, the stability always remains the key factor of concern owing to its importance in designing a neural networks, and such a situation motivates our present study. Thus, it is not only theoretically interesting but also practical to establish sufficient conditions for stability of neural networks with proportional delays.

Motivated by the above discussion, in this paper, we will investigate the problem of the input-to-state stability analysis for a class of the stochastic recurrent neural networks with multi-proportional delays. The given system here includes a stochastic Hopfield neural works with multi-proportional delays and stochastic cellular neural works with multi-proportional delays as its special forms. In addition, the stability criterion is the mean-square exponential input-to-state stability, which is more general than the traditionally mean-square exponential stability. By employing Lyapunov-Krasovskii functional, stochastic analysis theory and Itô's formula, the stability criterion is obtained. In addition, several examples and their simulations are given to guarantee the correctness and effectiveness of obtained results.

The rest of this paper is proposed as follows. In section 2, we introduce the mathematical model assumptions and definitions. In section 3, the main result and its proofs are provided. Section 4 propose several examples and their simulations to guarantee the correctness of the theoretical results. Conclusions are presented in Section 5. 


\section{Model description and preliminaries}

Let $C\left([q, 1] ; \mathbb{R}^{n}\right)$ denote the family of continuous functions $\phi$ from $[q, 1]$ to $\mathbb{R}^{n}$ with the uniform norm $\|\phi\|=\sup _{q \leq \theta \leq 1}|\phi(\theta)|$. Denote by $L_{\mathcal{F}_{0}}^{2}\left([q, 1] ; \mathbb{R}^{n}\right)$ the family of all $\mathcal{F}_{0}$ measurable, $C\left([q, 1] ; \mathbb{R}^{n}\right)$-valued stochastic variables $\phi=$ $\phi(s): q \leq s \leq 1$ such that $\int_{q}^{1} \mathbf{E}|\phi(s)|^{2} \mathrm{~d} s<\infty ; C\left([-\tau, 0] ; \mathbb{R}^{n}\right)$-valued stochastic variables $\phi=\phi(s):-\tau \leq s \leq 0$ such that $\int_{-\tau}^{0} \mathbf{E}|\phi(s)|^{2} \mathrm{~d} s<\infty$, in which $\mathbf{E}[\cdot]$ stands for the correspondent expectation operator with respect to the given probability measure $P . \ell_{\infty}$ denotes the class of essentially bounded functions $u$ from $[1, \infty)$ to $\mathbb{R}^{n}$ with $\|u\|_{\infty}=$ ess $\sup _{t \geq 1}|u(t)|<\infty$. $\mathbb{R}$ denotes real number. $\mathbb{R}^{n}$ denotes $\mathrm{n}$ dimensions Euclidean space.

In this paper, we discuss the following class of stochastic recurrent neural networks with multi-proportional delays:

$$
\begin{aligned}
\mathrm{d} x_{i}(t)=[ & -d_{i} x_{i}(t)+\sum_{j=1}^{n} a_{i j} f_{j}\left(x_{j}(t)\right)+\sum_{j=1}^{n} b_{i j} g_{j}\left(x_{j}\left(p_{j} t\right)\right)+\sum_{j=1}^{n} c_{i j} h_{j}\left(x_{j}\left(q_{j} t\right)\right) \\
& \left.+u_{i}(t)\right] \mathrm{d} t+\sum_{j=1}^{n} \sigma_{i j}\left(x_{j}(t), x_{j}\left(p_{j} t\right), x_{j}\left(q_{j} t\right)\right) \mathrm{d} w_{j}(t) \\
x_{i}(s)= & \varphi_{i}(t), t \in[q, 1]
\end{aligned}
$$

for all $t \geq 1, i=1,2, \cdots, n$, where $x_{i}(t)$ is the state variable of the $i$ th neuron at time $t, d_{i}$ is the self-feedback connection weight strength. The constants $a_{i j}, b_{i j}$ and $c_{i j}$ are the connection weights of the $j$ th neuron to the $i$ th neuron at time $t, p_{j} t$ and $q_{j} t$, respectively. $f_{j}(\cdot), g_{j}(\cdot)$ and $h_{j}(\cdot)$ are the $j$ th neuron activation functions at time $t, p_{j} t$ and $q_{j} t$, respectively. $u_{i}(t)$ is the control input of the $i$ th neuron at time $t$, and $u=\left(u_{1}(t), u_{2}(t), \cdots, u_{n}(t)\right) \in \ell_{\infty}$. The noise perturbation $\sigma_{i j}: \mathbb{R} \times \mathbb{R} \times \mathbb{R} \rightarrow \mathbb{R}$ is a Borel measurable function, and $w_{j}(t), j=1,2, \cdots, n$ are scalar standard Brownian motions defined on a complete probability space $(\Omega, \mathcal{F}, P)$ with a natural filtration $\left\{\mathcal{F}_{t}\right\}_{t \geq 1}$. The constants $p_{j}, q_{j}$ are proportional delay factors and satisfy $0<p_{j}, q_{j} \leq 1$, $p_{j} t=t-\left(1-p_{j}\right) t$ and $q_{j} t=t-\left(1-q_{j}\right) t$, where $\left(1-p_{j}\right) t$ and $\left(1-q_{j}\right) t$ are timevarying continuous functions that satisfy $\left(1-p_{j}\right) t \rightarrow+\infty$ and $\left(1-q_{j}\right) t \rightarrow+\infty$ as $t \rightarrow+\infty$ and $p_{j} \neq 1, q_{j} \neq 1 . q=\min _{1 \leq j \leq n}\left\{p_{j}, q_{j}\right\} . \varphi_{i}(t) \in C([q, 1] ; \mathbb{R})$, $\varphi(t)=\left(\varphi_{1}(t), \varphi_{2}(t), \cdots, \varphi_{n}(t)\right)^{T} \in C\left([q, 1] ; \mathbb{R}^{n}\right)$.

Let $\kappa$ denote the class of continuous strictly increasing functions $\varphi$ from $[0, \infty]$ to $[0, \infty]$ with $\varphi(0)=0$, and $\kappa \kappa$ denote the class of functions $\psi$ from $[0, \infty] \times[0, \infty]$ to $[0, \infty]$ satisfy:(i) the mapping $\varphi(t, \cdot)$ belongs to $\kappa$, for each fixed $t$,; (ii) the function $\psi(t, u)$ is decreasing to zero on $t$ as $t \rightarrow \infty$, for each fixed $u$.

The following assumptions will play an important part during the proof of main result of this paper. 
$\left(H_{1}\right)$ The activation functions $f_{i}(\cdot), g_{i}(\cdot)$ and $h_{i}(\cdot)$ satisfy Lipschitz condition, i.e. there exist positive constants $L_{i}, M_{i}$ and $N_{i}$ such that

$f_{i}(u)-f_{i}(v) \leq L_{i}|u-v|, g_{i}(u)-g_{i}(v) \leq M_{i}|u-v|, h_{i}(u)-h_{i}(v) \leq N_{i}|u-v|$,

for all $u, v \in \mathbb{R}, i=1,2, \cdots, n$.

$\left(H_{2}\right)$ For all $u, u^{\prime}, v, v^{\prime}, z, z^{\prime} \in \mathbb{R}, i, j=1,2, \cdots, n$, the noise perturbation $\sigma_{i j}$ in (2.1)-(2.2) satisfy

$$
\left[\sigma_{i j}(u, v, z)-\sigma_{i j}\left(u^{\prime}, v^{\prime}, z^{\prime}\right)\right]^{2} \leq \mu_{i j}\left(u-u^{\prime}\right)^{2}+\gamma_{i j}\left(v-v^{\prime}\right)^{2}+\delta_{i j}\left(z-z^{\prime}\right)^{2},
$$

where $\mu_{i j}, \gamma_{i j}$ and $\delta_{i j}$ are nonnegative constants.

$$
\left(H_{3}\right) f_{j}(0)=g_{j}(0)=h_{j}(0)=u_{j}(0)=0, \sigma_{i j}(0,0,0)=0, i, j=1,2, \cdots, n .
$$

Under $\left(H_{1}\right)-\left(H_{2}\right)$, it is easy to verify that the linear growth conditions hold, thereby there exists a unique solution of systems $(2.1)-(2.2)$. Let $x(t, \varphi)$ denote the solution from the initial data $x(s)=\varphi(s)$ on $s \in[q, 1]$ in $L_{\mathcal{F}_{0}}^{2}\left([q, 1] ; \mathbb{R}^{n}\right)$. It is obvious that under $\left(H_{3}\right)$, systems (2.1)-(2.2) own a trivial solution or zero solution $x(t ; 0) \equiv 0$ corresponding to the initial data $\varphi(s)=0$.

Then applying the following variable transformations $y_{i}(t)=x_{i}\left(\mathrm{e}^{t}\right), v_{i}(t)=$ $u_{i}\left(\mathrm{e}^{t}\right), \tilde{w}_{j}(t)=w_{j}\left(\mathrm{e}^{t}\right), \phi_{i}(t)=\varphi_{i}\left(\mathrm{e}^{t}\right)$, systems (2.1)-(2.2) are equivalently transformed into the following stochastic recurrent neural networks with unequal constant delays and time-varying coefficients (see [30])

$$
\begin{aligned}
\mathrm{d} y_{i}(t)= & \mathrm{e}^{t}\left\{-d_{i} y_{i}(t)+\sum_{j=1}^{n}\left[a_{i j} f_{j}\left(y_{j}(t)\right)+b_{i j} g_{j}\left(y_{j}\left(t-\tau_{j}\right)\right)+c_{i j} h_{j}\left(y_{j}\left(t-\zeta_{j}\right)\right)\right]\right. \\
& \left.+v_{i}(t)\right\} \mathrm{d} t+\mathrm{e}^{t}\left[\sum_{j=1}^{n} \sigma_{i j}\left(y_{j}(t), y_{j}\left(t-\tau_{j}\right), y_{j}\left(t-\zeta_{j}\right)\right)\right] \mathrm{d} \tilde{w}_{j} t \\
y_{i}(t)= & \phi_{i}(t), t \in[-\tau, 0] .
\end{aligned}
$$

where $t \geq 0, \tau_{j}=-\log p_{j}, \zeta_{j}=-\log q_{j}, \tau=\max _{1 \leq j \leq n}\left\{\tau_{j}, \zeta_{j}\right\}, j=1,2, \cdots, n, \phi_{i}(t) \in$ $C([-\tau, 0] ; \mathbb{R}), \phi(t)=\left(\phi_{1}(t), \phi_{2}(t), \cdots, \phi_{n}(t)\right)^{T} \in C\left([-\tau, 0] ; \mathbb{R}^{n}\right)$.

Remark 2.1 Systems (2.1)-(2.2) and (2.3)-(2.4) are equivalent, hence, if we investigate the mean-square exponentially input-to-state stability of systems (2.1)-(2.2), we can equivalently investigate systems (2.3)-(2.4).

Remark 2.2 Systems (2.1)-(2.2) and (2.3)-(2.4) are different from system in [23]. From the view of delays, the one in [23] are constant and bounded varying, while in (2.1)-(2.2) are unbounded time-varying. From the view of coefficient, the one in [23] are constant, while the other one in (2.3)-(2.4) which contains $\mathrm{e}^{\mathrm{t}}$ is time-varying. 
Next, we will propose two definitions of the input-to-state stability of systems (2.3)-(2.4).

Definition 2.3 [23] The trivial solution of systems (2.3)-(2.4) is said to be mean-square asymptotically input-to-state stable if for every $\phi \in L_{\mathcal{F}_{0}}^{2}\left([-\tau, 0] ; \mathbb{R}^{n}\right)$ and $u \in \ell_{\infty}$, there exist two functions $\varphi \in \kappa$ and $\psi \in \kappa \kappa$ such that:

$$
\mathbf{E}|x(t ; \phi)|^{2} \leq \psi\left(t, \mathbf{E}\|\phi\|^{2}\right)+\varphi\left(\|u\|_{\infty}^{2}\right) .
$$

Definition 2.4 [23]The trivial solution of systems (2.3)-(2.4) is said to be mean-square exponentially input-to-state stable if for every $\phi \in L_{\mathcal{F}_{0}}^{2}\left([-\tau, 0] ; \mathbb{R}^{n}\right)$ and $u \in \ell_{\infty}$, there exist scalars $\alpha>0, \beta>0$ and $\gamma>0$ such that:

$$
\mathbf{E}|x(t ; \phi)| \leq \alpha \mathrm{e}^{-\beta t} \mathbf{E}|| \phi\left\|^{2}+\gamma\right\| u \|_{\infty}^{2} .
$$

\section{$3 \quad$ Main results}

In this section, we will discuss the mean-square exponential input-to-state stability of the trivial solution for systems $(2.3)-(2.4)$ under $\left(H_{1}\right)-\left(H_{3}\right)$.

Theorem 3.1 Under $\left(H_{1}\right)-\left(H_{3}\right)$, the trivial solution of systems (2.3)-(2.4) is mean-square exponentially input-to-state stable, if there exist positive scalars $\eta_{i}, \alpha_{i}, \beta_{i}(i=1,2, \cdots, n), \lambda>1$ such that:

$$
\begin{gathered}
2 \eta_{i} d_{i} \geq(1+\lambda) \eta_{i}+\alpha_{i}+\beta_{i}+\sum_{j=1}^{n} \eta_{j} \mu j i+\sum_{j=1}^{n} \eta_{j}\left|a_{j i}\right| L_{i} \\
+\eta_{i} \sum_{j=1}^{n}\left|a_{i j}\right| L_{j}+\eta_{i} \sum_{j=1}^{n}\left|b_{i j}\right| M_{j}+\eta_{i} \sum_{j=1}^{n}\left|c_{i j}\right| N_{j} \\
\alpha_{i} \geq \sum_{j=1}^{n} \mathrm{e}^{\lambda \tau} \eta_{j}\left|b_{j i}\right| M_{i}+\sum_{j=1}^{n} \mathrm{e}^{\lambda \tau} \eta_{j} \gamma_{j i} \\
\beta_{i} \geq \sum_{j=1}^{n} \mathrm{e}^{\lambda \tau} \eta_{j}\left|c_{j i}\right| N_{i}+\sum_{j=1}^{n} \mathrm{e}^{\lambda \tau} \eta_{j} \delta_{j i}
\end{gathered}
$$

Proof. For the purpose of simplicity, we write $y(t ; \phi)=y(t)$ and denote $\sigma(t)=\left(\sigma_{i j}(t)\right)_{n \times n}$, where $\sigma_{i j}=\sigma_{i j}\left(y_{j}(t), y_{j}\left(t-\tau_{j}\right), y_{j}\left(t-\zeta_{j}\right)\right.$. Construct the following Lyapunov-Krasovskii functional:

$$
V(t, y(t))=\mathrm{e}^{(\lambda-1) t} \sum_{i=1}^{n} \eta_{i} y_{i}^{2}(t)+\int_{t-\tau_{i}}^{t} \mathrm{e}^{\lambda s} \sum_{i=1}^{n} \alpha_{i} y_{i}^{2}(s) \mathrm{d} s+\int_{t-\zeta_{i}}^{t} \mathrm{e}^{\lambda s} \sum_{i=1}^{n} \beta_{i} y_{i}^{2}(s) \mathrm{d} s .
$$


Then by Itô's formula, we have the following stochastic differential equation:

$$
\mathrm{d} V(t, y(t))=\mathcal{L} V(t, y(t)) \mathrm{d} t+V_{y}(t, y(t)) \sigma(t) \mathrm{d} w(t)
$$

where $V_{y}(t, y(t))=\left(\frac{\partial V((t, y(t)))}{\partial y_{1}}, \cdots, \frac{\partial V((t, y(t)))}{\partial y_{n}}\right)$, and $\mathcal{L}$ is the weak infinitesimal operator such that

$$
\begin{aligned}
& \mathcal{L} V(t, y(t))=(\lambda-1) \mathrm{e}^{(\lambda-1) t} \sum_{i=1}^{n} \eta_{i} y_{i}^{2}(t)+2 \mathrm{e}^{(\lambda-1) t} \sum_{i=1}^{n} \eta_{i} y_{i}(t) \mathrm{e}^{\lambda t}\left[-d_{i} y_{i}(t)+\right. \\
& \sum_{j=1}^{n} a_{i j} f_{j}\left(y_{j}(t)\right)+\sum_{j=1}^{n} b_{i j} g_{j}\left(y_{j}\left(t-\tau_{j}\right)\right)+\sum_{j=1}^{n} c_{i j} h_{j}\left(y_{j}\left(t-\zeta_{j}\right)\right)+ \\
& \left.\left.v_{i}(t)\right]+\mathrm{e}^{(\lambda-1) t} \sum_{i=1}^{n} \eta_{i} \sum_{j=1}^{n} \sigma_{i j}^{2}\left(y_{j}(t), y_{j}\left(t-\tau_{j}\right), y_{j}\left(t-\zeta_{j}\right)\right)\right)+ \\
& \mathrm{e}^{\lambda t} \sum_{i=1}^{n} \alpha_{i} y_{i}^{2}(t)-\mathrm{e}^{\lambda\left(t-\tau_{j}\right)} \sum_{i=1}^{n} \alpha_{i} y_{i}^{2}\left(t-\tau_{i}\right)+ \\
& \mathrm{e}^{\lambda t} \sum_{i=1}^{n} \beta_{i} y_{i}^{2}(t)-\mathrm{e}^{\lambda\left(t-\zeta_{j}\right)} \sum_{i=1}^{n} \beta_{i} y_{i}^{2}\left(t-\zeta_{i}\right)+ \\
& \leq \lambda \mathrm{e}^{\lambda t} \sum_{i=1}^{n} \eta_{i} y_{i}^{2}(t)-2 \mathrm{e}^{\lambda t} \sum_{i=1}^{n} \eta_{i} d_{i} y_{i}^{2}(t)+2 \mathrm{e}^{\lambda t} \sum_{i=1}^{n} \eta_{i} y_{i}(t) \sum_{j=1}^{n} a_{i j} f_{j}\left(y_{j}(t)\right)+ \\
& 2 \mathrm{e}^{\lambda t} \sum_{i=1}^{n} \eta_{i} y_{i}(t) \sum_{j=1}^{n} b_{i j} g_{j}\left(y_{j}\left(t-\tau_{j}\right)\right)+2 \mathrm{e}^{\lambda t} \sum_{i=1}^{n} \eta_{i} y_{i}(t) \sum_{j=1}^{n} c_{i j} h_{j}\left(y_{j}\left(t-\zeta_{j}\right)\right)+ \\
& 2 \mathrm{e}^{\lambda t} \sum_{i=1}^{n} \eta_{i} y_{i}(t) v_{i}(t)+\mathrm{e}^{\lambda t} \sum_{i=1}^{n} \eta_{i} \sum_{j=1}^{n} \mu_{i j} y_{j}^{2}(t)+\mathrm{e}^{\lambda t} \sum_{i=1}^{n} \eta_{i} \sum_{j=1}^{n} \gamma_{i j} y_{j}^{2}\left(t-\tau_{j}\right)+ \\
& \mathrm{e}^{\lambda t} \sum_{i=1}^{n} \eta_{i} \sum_{j=1}^{n} \delta_{i j} y_{j}^{2}\left(t-\zeta_{j}\right)+\mathrm{e}^{\lambda t} \sum_{i=1}^{n} \alpha_{i} y_{i}^{2}(t)-\mathrm{e}^{\lambda(t-\tau)} \sum_{i=1}^{n} \alpha_{i} y_{i}^{2}\left(t-\tau_{i}\right)+ \\
& \mathrm{e}^{\lambda t} \sum_{i=1}^{n} \beta_{i} y_{i}^{2}(t)-\mathrm{e}^{\lambda(t-\tau)} \sum_{i=1}^{n} \beta_{i} y_{i}^{2}\left(t-\zeta_{i}\right) \\
& \leq \mathrm{e}^{\lambda t} \sum_{i=1}^{n}\left(-2 \eta_{i} d_{i}+\lambda \eta_{i}+\sum_{j=1}^{n} \eta_{j} \mu_{j i}+\alpha_{i}+\beta_{i}\right) y_{i}^{2}(t)+ \\
& 2 \mathrm{e}^{\lambda t} \sum_{i=1}^{n} \eta_{i}\left|y_{i}(t)\right| \sum_{j=1}^{n}\left|a_{i j}\right|\left|f_{j}\left(y_{j}(t)\right)-f_{j}(0)\right|+ \\
& 2 \mathrm{e}^{\lambda t} \sum_{i=1}^{n} \eta_{i}\left|y_{i}(t)\right| \sum_{j=1}^{n}\left|b_{i j}\right|\left|g_{j}\left(y_{j}\left(t-\tau_{j}\right)\right)-g_{j}(0)\right|+ \\
& 2 \mathrm{e}^{\lambda t} \sum_{i=1}^{n} \eta_{i}\left|y_{i}(t)\right| \sum_{j=1}^{n}\left|c_{i j}\right|\left|h_{j}\left(y_{j}\left(t-\zeta_{j}\right)\right)-h_{j}(0)\right|+
\end{aligned}
$$




$$
\begin{aligned}
& 2 \mathrm{e}^{\lambda t} \sum_{i=1}^{n} \eta_{i}\left|y_{i}(t)\right|\left|v_{i}(t)\right|+\mathrm{e}^{\lambda t} \sum_{i=1}^{n}\left(-\mathrm{e}^{-\lambda \tau} \alpha_{i}+\sum_{j=1}^{n} \eta_{j} \gamma_{j i}\right) y_{i}^{2}\left(t-\tau_{i}\right)+ \\
& \mathrm{e}^{\lambda t} \sum_{i=1}^{n}\left(-\mathrm{e}^{\lambda \tau} \beta_{i}+\sum_{j=1}^{n} \eta_{j} \delta_{j i}\right) y_{i}^{2}\left(t-\zeta_{i}\right) \\
& \leq \mathrm{e}^{\lambda t} \sum_{i=1}^{n}\left(-2 \eta_{i} d_{i}+\lambda \eta_{i}+\sum_{j=1}^{n} \eta_{j} \mu_{j i}+\alpha_{i}+\beta_{i}\right) y_{i}^{2}(t)+ \\
& 2 \mathrm{e}^{\lambda t} \sum_{i=1}^{n} \eta_{i}\left|y_{i}(t)\right| \sum_{j=1}^{n}\left|a_{i j}\right| L_{j}\left|y_{j}(t)\right|+2 \mathrm{e}^{\lambda t} \sum_{i=1}^{n} \eta_{i}\left|y_{i}(t)\right| \sum_{j=1}^{n}\left|b_{i j}\right| M_{j}\left|y_{j}\left(t-\tau_{j}\right)\right|+ \\
& 2 \mathrm{e}^{\lambda t} \sum_{i=1}^{n} \eta_{i}\left|y_{i}(t)\right| \sum_{j=1}^{n}\left|c_{i j}\right| N_{j}\left|y_{j}\left(t-\zeta_{j}\right)\right|+2 \mathrm{e}^{\lambda t} \sum_{i=1}^{n} \eta_{i}\left|y_{i}(t)\right|\left|v_{i}(t)\right|+ \\
& \mathrm{e}^{\lambda t} \sum_{i=1}^{n}\left(-\mathrm{e}^{-\lambda \tau} \alpha_{i}+\sum_{j=1}^{n} \eta_{j} \gamma_{j i}\right) y_{i}^{2}\left(t-\tau_{i}\right)+ \\
& \mathrm{e}^{\lambda t} \sum_{i=1}^{n}\left(-\mathrm{e}^{\lambda \tau} \beta_{i}+\sum_{j=1}^{n} \eta_{j} \delta_{j i}\right) y_{i}^{2}\left(t-\zeta_{i}\right) \\
& \leq \mathrm{e}^{\lambda t} \sum_{i=1}^{n}\left(-2 \eta_{i} d_{i}+\lambda \eta_{i}+\sum_{j=1}^{n} \eta_{j} \mu_{j i}+\alpha_{i}+\beta_{i}\right) y_{i}^{2}(t)+ \\
& \mathrm{e}^{\lambda t} \sum_{i=1}^{n} \sum_{j=1}^{n} \eta_{i}\left|a_{i j}\right| L_{j}\left(\left|y_{i}(t)\right|^{2}+\left|y_{j}(t)\right|^{2}\right)+ \\
& \mathrm{e}^{\lambda t} \sum_{i=1}^{n} \sum_{j=1}^{n} \eta_{i}\left|b_{i j}\right| M_{j}\left(\left|y_{i}(t)\right|^{2}+\left|y_{j}\left(t-\tau_{j}\right)\right|^{2}\right)+ \\
& \mathrm{e}^{\lambda t} \sum_{i=1}^{n} \sum_{j=1}^{n} \eta_{i}\left|c_{i j}\right| N_{j}\left(\left|y_{i}(t)\right|^{2}+\left|y_{j}\left(t-\zeta_{j}\right)\right|^{2}\right)+ \\
& \mathrm{e}^{\lambda t} \sum_{i=1}^{n} \eta_{i}\left(\left|y_{i}(t)\right|^{2}+\left|v_{i}(t)\right|^{2}\right)+\mathrm{e}^{\lambda t} \sum_{i=1}^{n}\left(-\mathrm{e}^{-\lambda \tau} \alpha_{i}+\sum_{j=1}^{n} \eta_{j} \gamma_{j i}\right) y_{i}^{2}\left(t-\tau_{i}\right)+ \\
& \mathrm{e}^{\lambda t} \sum_{i=1}^{n}\left(-\mathrm{e}^{\lambda \tau} \beta_{i}+\sum_{j=1}^{n} \eta_{j} \delta_{j i}\right) y_{i}^{2}\left(t-\zeta_{i}\right) \\
& \leq-\mathrm{e}^{\lambda t} \min _{1 \leq j \leq n}\left[2 \eta_{j} d_{j}-\lambda \eta_{j}-\sum_{k=1}^{n} \eta_{k} \mu_{k j}-\alpha_{j}-\beta_{j}-\eta_{j} \sum_{k=1}^{n}\left|a_{j k}\right| L_{k}-\right. \\
& \left.\sum_{k=1}^{n} \eta_{k}\left|a_{k j}\right| L_{j}-\sum_{k=1}^{n} \eta_{j}\left|b_{j k}\right| M_{k}-\sum_{k=1}^{n} \eta_{j}\left|c_{j k}\right| N_{k}-\eta_{j}\right] \sum_{i=1}^{n} y_{i}^{2}(t)- \\
& \mathrm{e}^{\lambda t} \min _{1 \leq j \leq n}\left[\mathrm{e}^{\lambda \tau} \alpha_{j}-\sum_{k=1}^{n} \eta_{k} \gamma_{k j}-\sum_{k=1}^{n} \eta_{k}\left|b_{k j}\right| M_{j}\right] \sum_{j=1}^{n} y_{i}^{2}\left(t-\tau_{i}\right)- \\
& \mathrm{e}^{\lambda t} \min _{1 \leq j \leq n}\left[\mathrm{e}^{\lambda \tau} \beta_{j}-\sum_{k=1}^{n} \eta_{k} \delta_{k j}-\sum_{k=1}^{n} \eta_{k}\left|c_{k j}\right| N_{j}\right] \sum_{j=1}^{n} y_{i}^{2}\left(t-\zeta_{i}\right)+ \\
& \mathrm{e}^{\lambda t} \sum_{i=1}^{n} \eta_{i}\left|v_{i}(t)\right|^{2}
\end{aligned}
$$




$$
\begin{aligned}
\leq & -\mathrm{e}^{\lambda t} \min _{1 \leq j \leq n}\left[2 \eta_{j} d_{j}-\lambda \eta_{j}-\sum_{k=1}^{n} \eta_{k} \mu_{k j}-\alpha_{j}-\beta_{j}-\eta_{j} \sum_{k=1}^{n}\left|a_{j k}\right| L_{k}-\right. \\
& \left.\sum_{k=1}^{n} \eta_{k}\left|a_{k j}\right| L_{j}-\sum_{k=1}^{n} \eta_{j}\left|b_{j k}\right| M_{k}-\sum_{k=1}^{n} \eta_{j}\left|c_{j k}\right| N_{k}-\eta_{j}\right] \sum_{i=1}^{n} y_{i}^{2}(t)- \\
& \mathrm{e}^{\lambda t} \min _{1 \leq j \leq n}\left[\mathrm{e}^{-\lambda \tau} \alpha_{j}-\sum_{k=1}^{n} \eta_{k} \gamma_{k j}-\sum_{k=1}^{n} \eta_{k}\left|b_{k j}\right| M_{j}\right] \sum_{j=1}^{n} y_{i}^{2}\left(t-\tau_{i}\right)- \\
& \mathrm{e}^{\lambda t} \min _{1 \leq j \leq n}\left[\mathrm{e}^{-\lambda \tau} \beta_{j}-\sum_{k=1}^{n} \eta_{k} \delta_{k j}-\sum_{k=1}^{n} \eta_{k}\left|c_{k j}\right| N_{j}\right] \sum_{j=1}^{n} y_{i}^{2}\left(t-\zeta_{i}\right)+ \\
& \mathrm{e}^{\lambda t} \max _{1 \leq j \leq n} \eta_{j}|| v \|_{\infty}^{2} .
\end{aligned}
$$

Now, we define a Markov time as follows:

$$
\rho_{k}:=\inf \{s \geq 0:|x(s)| \geq k\}
$$

By means of the Dynkin formula, we have

$$
\mathbf{E} V\left(t \wedge \rho_{k}, y\left(t \wedge \rho_{k}\right)\right)-\mathbf{E} V(0, y(0))=\mathbf{E}\left[\int_{0}^{t \wedge \rho_{k}} \mathcal{L} V(s, y(s)) \mathrm{d} s\right]
$$

which implies

$$
\mathbf{E} V\left(t \wedge \rho_{k}, y\left(t \wedge \rho_{k}\right)\right)=\mathbf{E} V(0, y(0))+\mathbf{E}\left[\int_{0}^{t \wedge \rho_{k}} \mathcal{L} V(s, y(s)) \mathrm{d} s\right]
$$

Letting $k \rightarrow \infty$ on both sides (3.5), it follows from the monotone convergence theorem, (3.1)-(3.3) and (3.4) that

$$
\begin{aligned}
\mathbf{E} V(t, y(t)) \leq & \mathbf{E} V(0, x(0))+\|v\|_{\infty}^{2} \max _{1 \leq j \leq n} \eta_{j} \int_{0}^{t} \mathrm{e}^{\lambda s} \mathrm{~d} s \\
\leq & \mathbf{E} V(0, x(0))+\frac{1}{\lambda}\|v\|_{\infty}^{2} \max _{1 \leq j \leq n} \eta_{j}\left(\mathrm{e}^{\lambda t}-1\right) \\
= & \sum_{i=1}^{n} \eta_{i} \mathbf{E} y_{i}^{2}(0)+\int_{-\tau_{i}}^{0} \mathbf{E e}^{\lambda s} \sum_{i=1}^{n} \alpha_{i} y_{i}^{2}(s) \mathrm{d} s+ \\
& \int_{-\zeta_{i}}^{0} \mathbf{E e}^{\lambda s} \sum_{i=1}^{n} \beta_{i} y_{i}^{2}(s) \mathrm{d} s+\frac{1}{\lambda}\|v\|_{\infty}^{2} \max _{1 \leq j \leq n} \eta_{j}\left(\mathrm{e}^{\lambda t}-1\right) \\
\leq & \left(\max _{1 \leq j \leq n} \eta_{j}+\tau \max _{1 \leq j \leq n} \alpha_{j}+\tau \max _{1 \leq j \leq n} \beta_{j}\right) \mathbf{E}\|\varphi\|^{2}+ \\
& \frac{1}{\lambda}\|v\|_{\infty}^{2} \max _{1 \leq j \leq n} \eta_{j}\left(\mathrm{e}^{\lambda t}-1\right) .
\end{aligned}
$$

On the other hand, from the definition of $V(t, x(t))$, we can get that

$$
\mathbf{E} V(t, y(t)) \geq \mathbf{E e}^{(\lambda-1) t} \sum_{i=1}^{n} \eta_{i} y_{i}^{2}(t) \geq \mathrm{e}^{(\lambda-1) t} \min _{1 \leq i \leq n} \eta_{i} \mathbf{E}|x(t)|^{2} .
$$


Combining (3.6) and (3.7), the following inequation holds:

$$
\mathbf{E}|x(t)|^{2} \leq \frac{\max _{1 \leq i \leq n} \eta_{i}+\tau \max _{1 \leq i \leq n} \alpha_{i}+\tau \max _{1 \leq i \leq n} \beta_{i}}{\min _{1 \leq i \leq n} \eta_{i}} \mathrm{e}^{(\lambda-1) t} \mathbf{E}\|\phi\|^{2}+\frac{\max _{1 \leq i \leq n} \eta_{i}}{\lambda \max _{1 \leq i \leq n} \eta_{i}}\|v\|_{\infty}^{2}
$$

From the Definition 2.3 and (3.8), we derive that the trivial solution of system (2.3)-(2.4) is mean-square exponentially input-to-state stable. The proof of Theorem 3.1 is completed.

Theorem 3.2 Assume that all the conditions of Theorem 3.1 hold. Then the trivial solution of system (2.3)-(2.4) with $v(t) \equiv 0$ is mean-square exponentially stable.

Remark 3.3 If $p_{j}=q_{j}=1, j=1,2, \cdots, n$, system (2.1)-(2.2) becomes the stochastic recurrent neural network without delays. The results in this paper are also applicable to the stochastic recurrent neural networks without delays.

\section{Illustrative examples}

In this section, we will give several examples to show the effectiveness of the obtained result here.

Example 4.1 Consider the case of 2-dimensions stochastic recurrent neural networks with multi-proportional delays (Except for time delays, the other data are the same as [23])

$$
\begin{aligned}
d x_{i}(t)= & -d_{i} x_{i}(t)+\sum_{j=1}^{2} a_{i j} f_{j}\left(x_{j}(t)\right)+\sum_{j=1}^{2} b_{i j} g_{j}\left(x_{j}\left(p_{j} t\right)\right)+\sum_{j=1}^{2} c_{i j} h_{j}\left(x_{j}\left(q_{j} t\right)\right) \\
& \left.+u_{i}(t)\right] \mathrm{d} t+\sum_{j=1}^{2} \sigma_{i j}\left(x_{j}(t), x_{j}\left(p_{j} t\right), x_{j}\left(q_{j} t\right)\right) \mathrm{d} w_{j}(t), \\
x_{i}(s)= & \varphi_{i}(t), \quad t \in[q, 1], i=1,2,
\end{aligned}
$$

where

$$
f(x)=g(x)=h(x)=\left\{\begin{array}{ll}
0.2 x, & \text { if } x \leq 0, \\
0.2 \tan (x), & \text { if } x>0,
\end{array} u(t)=0.1 \sin (t)\right.
$$

and

$$
\left(\sigma_{i j}\left(x_{j}(t)\right), x_{j}\left(p_{j} t\right), x_{j}\left(q_{j} t\right)\right)_{2 \times 2}=\left(\begin{array}{cc}
0.5 x_{1}(t) & 0.3\left(x_{2}(t)+x_{2}\left(p_{2} t\right)\right) \\
0.4 x_{1}\left(p_{1} t\right) & 0.3\left(x_{2}(t)+x_{2}\left(q_{2} t\right)\right)
\end{array}\right) .
$$


Other parameters of systems (4.1)-(4.2) are given as follows:

$$
\left(a_{i j}\right)_{2 \times 2}=\left(\begin{array}{cc}
-0.8 & 1.4 \\
0.5 & -1.3
\end{array}\right),\left(b_{i j}\right)_{2 \times 2}=\left(\begin{array}{cc}
-2.1 & 1.2 \\
-2.3 & -0.8
\end{array}\right),\left(c_{i j}\right)_{2 \times 2}=\left(\begin{array}{ll}
0.9 & 0.8 \\
0.4 & 1.4
\end{array}\right),
$$

$d_{1}=8, d_{2}=7$. Take $p_{1}=p_{2}=q_{1}=q_{2}=0.5, \lambda=1.1, \eta_{1}=0.4, \eta_{2}=$ $0.4, \alpha_{1}=2.7, \alpha_{2}=1.4, \beta_{1}=1.2, \beta_{2}=1.8$, from the definition of $\tau$, we get that $\tau=0.6931$. It is easy to check that assumptions $\left(H_{1}\right)-\left(H_{3}\right)$ are satisfied. We obtain by

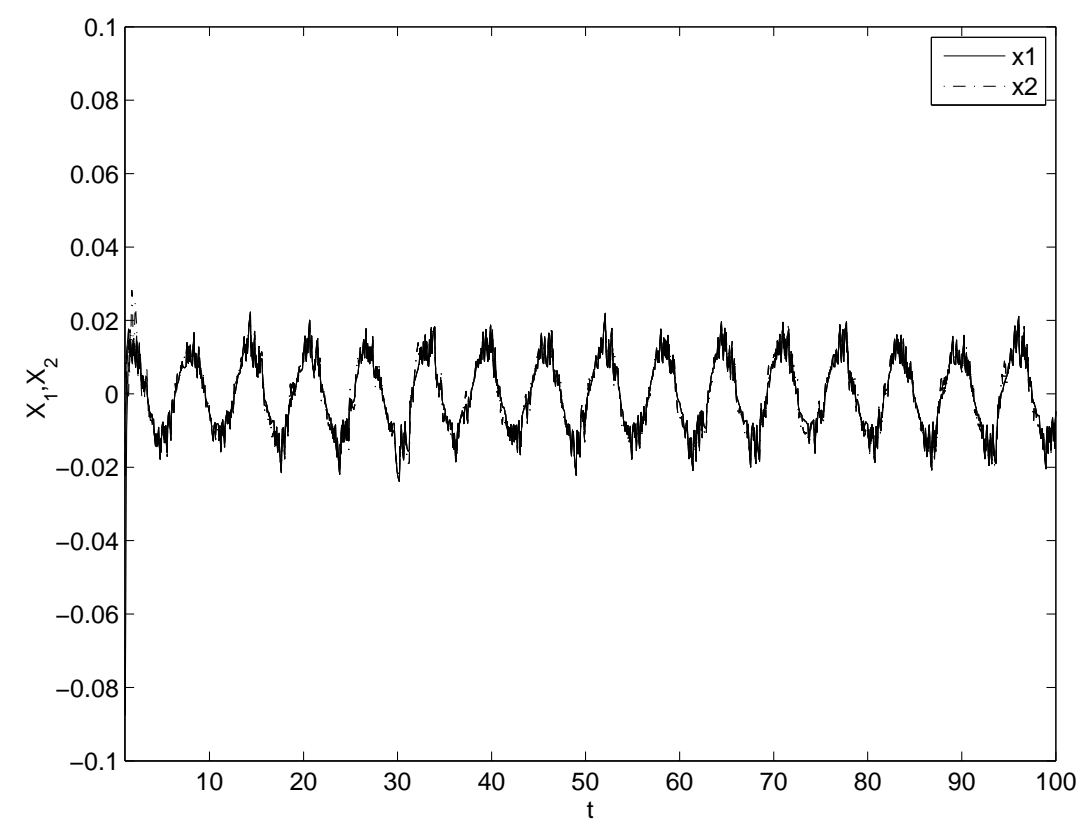

Fig. 1. The state responses of networks (4.1)-(4.2) in Example 4.1 with impulse in initial value $[0.6,-0.4]^{T},[0.2,-0.15]^{T}$ and $[0.02,-0.04]^{T}$.

$$
\begin{aligned}
2 \eta_{1} d_{1}= & 6.4000>(1+\lambda) \eta_{1}+\alpha_{1}+\beta_{1}+\sum_{j=1}^{2} \eta_{j} \mu_{j 1}+\sum_{j=1}^{2} \eta_{j}\left|a_{j 1}\right| L_{1}+ \\
& \eta_{1} \sum_{j=1}^{2}\left|a_{1 j}\right| L_{j}+\eta_{1} \sum_{j=1}^{2}\left|b_{1 j}\right| M_{j}+\eta_{1} \sum_{j=1}^{2}\left|c_{1 j}\right| N_{j}=5.6200, \\
2 \eta_{2} d_{2}= & 5.6000>(1+\lambda) \eta_{2}+\alpha_{2}+\beta_{2}+\sum_{j=1}^{2} \eta_{j} \mu_{j 2}+\sum_{j=1}^{2} \eta_{j}\left|a_{j 2}\right| L_{2}+ \\
& \eta_{2} \sum_{j=1}^{2}\left|a_{2 j}\right| L_{j}+\eta_{2} \sum_{j=1}^{2}\left|b_{2 j}\right| M_{j}+\eta_{2} \sum_{j=1}^{2}\left|c_{2 j}\right| N_{j}=5.0320,
\end{aligned}
$$




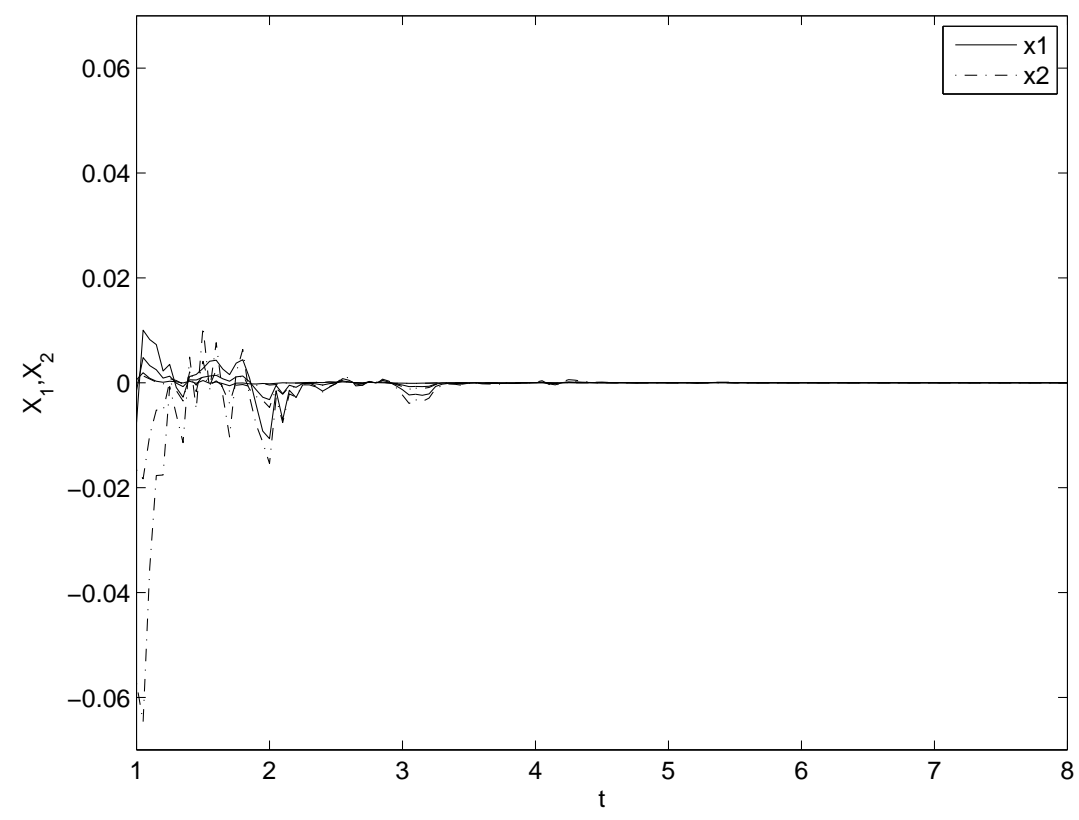

Fig. 2. The state responses of networks (4.1)-(4.2) with $u(t)=0$ in Example 4.1 with initial value $[0.6,-0.4]^{T},[0.2,-0.15]^{T}$ and $[0.02,-0.04]^{T}$.

$$
\begin{aligned}
& \alpha_{1}=2.7000>\sum_{j=1}^{2} \mathrm{e}^{\lambda \tau} \eta_{j}\left|b_{j 1}\right| M_{1}+\sum_{j=1}^{2} \mathrm{e}^{\lambda \tau} \eta_{j} \gamma_{j 1}=1.0975, \\
& \alpha_{2}=1.4000>\sum_{j=1}^{2} \mathrm{e}^{\lambda \tau} \eta_{j}\left|b_{j 2}\right| M_{2}+\sum_{j=1}^{2} \mathrm{e}^{\lambda \tau} \eta_{j} \gamma_{j 2}=0.6002, \\
& \beta_{1}=1.2000>\sum_{j=1}^{2} \mathrm{e}^{\lambda \tau} \eta_{j}\left|c_{j 1}\right| N_{1}+\sum_{j=1}^{2} \mathrm{e}^{\lambda \tau} \eta_{j} \delta_{j 1}=0.2229, \\
& \beta_{2}=1.8000>\sum_{j=1}^{2} \mathrm{e}^{\lambda \tau} \eta_{j}\left|c_{j 2}\right| N_{2}+\sum_{j=1}^{2} \mathrm{e}^{\lambda \tau} \eta_{j} \delta_{j 2}=0.6345 .
\end{aligned}
$$

Hence, all the conditions of Theorem 3.1 are satisfied. Systems (4.1)-(4.2) are mean-square exponentially input-to-state stable (See Fig. 1). Obviously, systems (4.1)-(4.2) are mean-square exponentially stable when $u(t) \equiv 0$, which can also be seen from Fig. 2.

Remark 4.2 In addition to the time delays term, Examples 4.1 in this paper, and Example 1 in [23] are the completely same. However, results in [23] are only suitable for constant and time-varying delays, results in this paper can applicable to unbounded proportional delays.Theorem 3.1 in this paper can applied in Example 1 in [23], but Theorem 1 in [23] can not applied in this paper. So Theorem 3.1 is more general than those in [23].

Example 4.3 (3-dimensions case). Consider the case of 3-dimensions stochas- 
tic recurrent neural networks with multi-proportional delays

$$
\begin{aligned}
d x_{i}(t)=[ & -d_{i} x_{i}(t)+\sum_{j=1}^{3} a_{i j} f_{j}\left(x_{j}(t)\right)+\sum_{j=1}^{3} b_{i j} g_{j}\left(x_{j}\left(p_{j} t\right)\right)+\sum_{j=1}^{3} c_{i j} h_{j}\left(x_{j}\left(q_{j} t\right)\right) \\
& \left.+u_{i}(t)\right] \mathrm{d} t+\sum_{j=1}^{3} \sigma_{i j}\left(x_{j}(t), x_{j}\left(p_{j} t\right), x_{j}\left(q_{j} t\right)\right) \mathrm{d} w_{j}(t) \\
x_{i}(s)= & \varphi_{i}(t), t \in[q, 1], i=1,2,3
\end{aligned}
$$

where

$$
\begin{gathered}
f(x)=g(x)=h(x)=0.3 \tanh (x), u(t)=0.2 \sin (t), \\
\left(\sigma_{i j}\left(x_{j}(t)\right), x_{j}\left(p_{j} t\right), x_{j}\left(q_{j} t\right)\right)_{3 \times 3}= \\
\left(\begin{array}{ccc}
0.2 x_{1}(t) & 0.3\left(x_{2}\left(p_{2} t\right)\right. & 0.3 x_{3}\left(q_{3} t\right) \\
0.5 x_{1}\left(p_{1} t\right) & 0.4\left(x_{2}(t)+x_{2}\left(q_{2} t\right)\right) & 0.1 x_{3}\left(p_{3} t\right) \\
0.3 x_{1}(t) & 0.2 x_{2}\left(q_{2} t\right) & 0.7\left(x_{3}(t)+x_{3}\left(p_{3}(t)\right)\right)
\end{array}\right) .
\end{gathered}
$$

Other parameters of system (4.3)-(4.4) are given as follows:

$$
\begin{gathered}
\left(a_{i j}\right)_{3 \times 3}=\left(\begin{array}{ccc}
-1 & 0.6 & 0.4 \\
1.3 & -0.4 & 0.7 \\
0.3 & 1.5 & -0.5
\end{array}\right), \quad\left(b_{i j}\right)_{3 \times 3}=\left(\begin{array}{ccc}
-0.8 & 0.2 & 0.5 \\
-0.7 & -1.3 & 0.6 \\
1.5 & -0.4 & 2.1
\end{array}\right), \\
\left(c_{i j}\right)_{3 \times 3}=\left(\begin{array}{ccc}
1.4 & -0.8 & 1.3 \\
0.4 & -0.1 & -0.5 \\
-1.7 & 0.5 & -0.7
\end{array}\right)
\end{gathered}
$$

$d_{1}=7, d_{2}=8, d_{3}=9$. Take $p_{1}=p_{2}=p_{3}=0.6 ; q_{1}=q_{2}=q_{3}=0.5, \lambda=$ $1.5, \eta_{1}=0.5, \eta_{2}=0.3, \eta_{3}=0.4, \alpha_{1}=1.6, \alpha_{2}=1.5, \alpha_{3}=1.9, \beta_{1}=$ $1.4, \beta_{2}=1.2, \beta_{3}=1.5$, from the definition of $\tau$, we get that $\tau=0.6931$. It is 
easy to check that assumptions $\left(H_{1}\right)-\left(H_{3}\right)$ are satisfied. We obtain by

$$
\begin{aligned}
2 \eta_{1} d_{1}= & 7.0000>(1+\lambda) \eta_{1}+\alpha_{1}+\beta_{1}+\sum_{j=1}^{3} \eta_{j} \mu_{j 1}+\sum_{j=1}^{3} \eta_{j}\left|a_{j 1}\right| L_{1}+ \\
& \eta_{1} \sum_{j=1}^{3}\left|a_{1 j}\right| L_{j}+\eta_{1} \sum_{j=1}^{3}\left|b_{1 j}\right| M_{j}+\eta_{1} \sum_{j=1}^{3}\left|c_{1 j}\right| N_{j}=5.8230, \\
2 \eta_{2} d_{2}= & 4.8000>(1+\lambda) \eta_{2}+\alpha_{2}+\beta_{2}+\sum_{j=1}^{3} \eta_{j} \mu_{j 2}+\sum_{j=1}^{3} \eta_{j}\left|a_{j 2}\right| L_{2}+ \\
& \eta_{2} \sum_{j=1}^{3}\left|a_{2 j}\right| L_{j}+\eta_{2} \sum_{j=1}^{3}\left|b_{2 j}\right| M_{j}+\eta_{2} \sum_{j=1}^{3}\left|c_{2 j}\right| N_{j}=4.4160, \\
2 \eta_{3} d_{3}= & 7.2000>(1+\lambda) \eta_{3}+\alpha_{3}+\beta_{3}+\sum_{j=1}^{3} \eta_{j} \mu_{j 3}+\sum_{j=1}^{3} \eta_{j}\left|a_{j 3}\right| L_{3}+ \\
& \eta_{3} \sum_{j=1}^{3}\left|a_{3 j}\right| L_{j}+\eta_{3} \sum_{j=1}^{3}\left|b_{3 j}\right| M_{j}+\eta_{3} \sum_{j=1}^{3}\left|c_{3 j}\right| N_{j}=5.9670,
\end{aligned}
$$

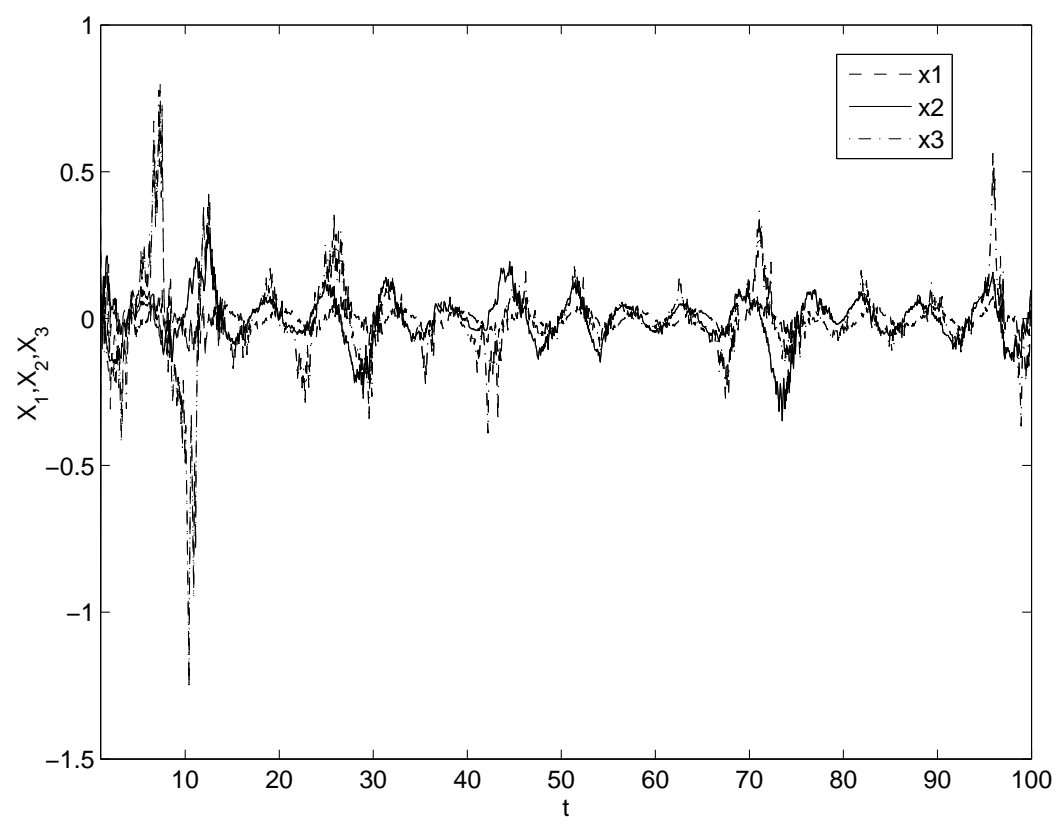

Fig. 3. The state responses of networks (4.3)-(4.4) with impulse in initial value $[0.3,-0.2,0.25]^{T}$ and $[0.4,-1,0.3]^{T}$. 


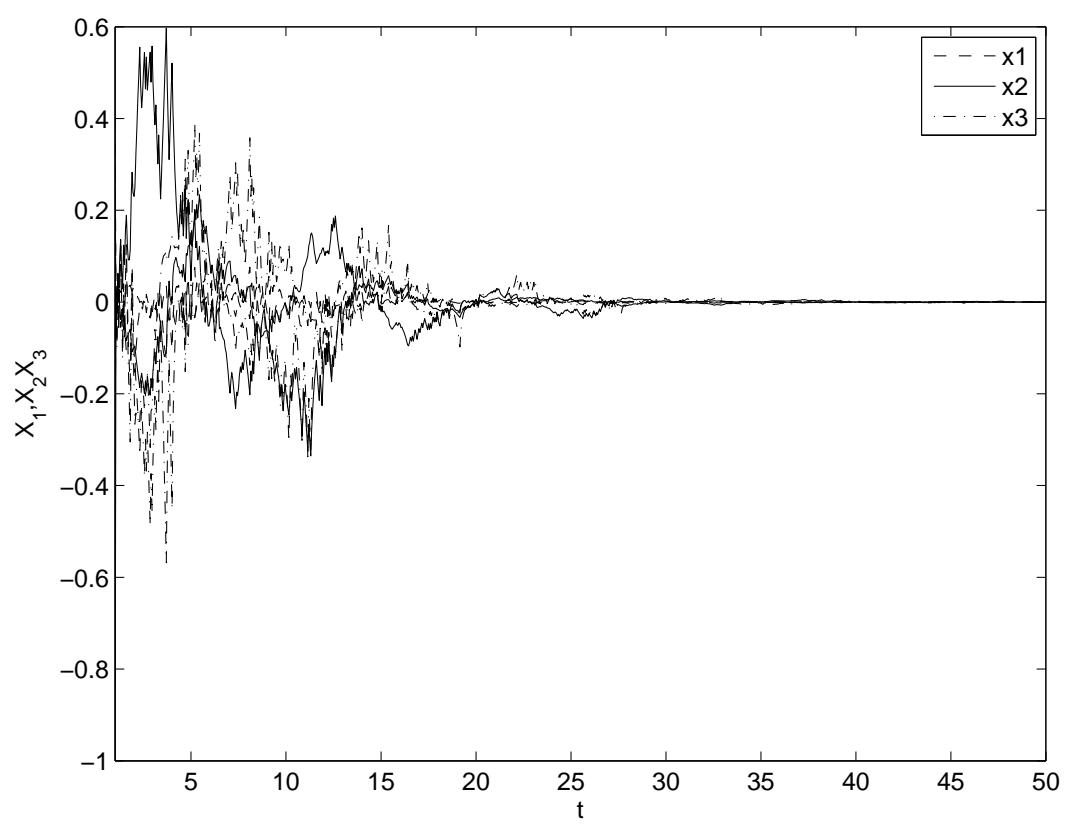

Fig. 4. The state responses of networks (4.3)-(4.4) with $u(t)=0$ with initial value $[0.3,-0.2,0.25]^{T}$ and $[0.4,-1,0.3]^{T}$.

$$
\begin{aligned}
& \alpha_{1}=1.6000>\sum_{j=1}^{3} \mathrm{e}^{\lambda \tau} \eta_{j}\left|b_{j 1}\right| M_{1}+\sum_{j=1}^{3} \mathrm{e}^{\lambda \tau} \eta_{j} \gamma_{j 1}=1.4509, \\
& \alpha_{2}=1.5000>\sum_{j=1}^{3} \mathrm{e}^{\lambda \tau} \eta_{j}\left|b_{j 2}\right| M_{2}+\sum_{j=1}^{3} \mathrm{e}^{\lambda \tau} \eta_{j} \gamma_{j 2}=1.3151, \\
& \alpha_{3}=1.9000>\sum_{j=1}^{3} \mathrm{e}^{\lambda \tau} \eta_{j}\left|b_{j 3}\right| M_{3}+\sum_{j=1}^{3} \mathrm{e}^{\lambda \tau} \eta_{j} \gamma_{j 3}=1.8695, \\
& \beta_{1}=1.4000>\sum_{j=1}^{3} \mathrm{e}^{\lambda \tau} \eta_{j}\left|c_{j 1}\right| N_{1}+\sum_{j=1}^{3} \mathrm{e}^{\lambda \tau} \eta_{j} \delta_{j 1}=1.2727, \\
& \beta_{2}=1.2000>\sum_{j=1}^{3} \mathrm{e}^{\lambda \tau} \eta_{j}\left|c_{j 2}\right| N_{2}+\sum_{j=1}^{3} \mathrm{e}^{\lambda \tau} \eta_{j} \delta_{j 2}=0.7608 \\
& \beta_{3}=1.5000>\sum_{j=1}^{3} \mathrm{e}^{\lambda \tau} \eta_{j}\left|c_{j 3}\right| N_{3}+\sum_{j=1}^{3} \mathrm{e}^{\lambda \tau} \eta_{j} \delta_{j 3}=1.4254 .
\end{aligned}
$$

Hence, all the conditions of Theorem 3.1 are satisfied. Systems (4.3)-(4.4) are mean-square exponentially input-to-state stable (See Fig. 3). Obviously. Systems (4.3)-(4.4) are mean-square exponentially stable when $u(t) \equiv 0$, which can also be seen from Fig. 4.

The Examples 4.1 and 4.3 can verify Theorem 3.1. Next, we will give a counter example to enrich our paper. 
Example 4.4 Consider the case of 2-dimensions stochastic recurrent neural networks with multi-proportional delays

$$
\begin{aligned}
d x_{i}(t)= & {\left[-d_{i} x_{i}(t)+\sum_{j=1}^{2} a_{i j} f_{j}\left(x_{j}(t)\right)+\sum_{j=1}^{2} b_{i j} g_{j}\left(x_{j}\left(p_{j} t\right)\right)+\sum_{j=1}^{2} c_{i j} h_{j}\left(x_{j}\left(q_{j} t\right)\right)\right.} \\
& \left.+u_{i}(t)\right] \mathrm{d} t+\sum_{j=1}^{2} \sigma_{i j}\left(x_{j}(t), x_{j}\left(p_{j} t\right), x_{j}\left(q_{j} t\right)\right) \mathrm{d} w_{j}(t) \\
x_{i}(s)= & \varphi_{i}(t), t \in[q, 1], i=1,2
\end{aligned}
$$

where

$$
f(x)=g(x)=h(x)=\sin (1 / t), u(t)=0.1 \sin (t)
$$

and

$$
\left(\sigma_{i j}\left(x_{j}(t)\right), x_{j}\left(p_{j} t\right), x_{j}\left(q_{j} t\right)\right)_{2 \times 2}=\left(\begin{array}{cc}
0.3 x_{1}(t) & 0.2\left(x_{2}(t)+0.2 x_{2}\left(p_{2} t\right)\right) \\
0.2 x_{1}\left(p_{1} t\right) & 0.3\left(x_{2}(t)+0.5 x_{2}\left(q_{2} t\right)\right)
\end{array}\right) .
$$

Other parameters of systems (4.1)-(4.2) are given as follows:

$$
\left(a_{i j}\right)_{2 \times 2}=\left(\begin{array}{cc}
-0.2 & 1.4 \\
0.3 & -1.5
\end{array}\right),\left(b_{i j}\right)_{2 \times 2}=\left(\begin{array}{cc}
-1.1 & 1.5 \\
-2.2 & -0.3
\end{array}\right),\left(c_{i j}\right)_{2 \times 2}=\left(\begin{array}{cc}
0.7 & 0.5 \\
0.8 & 1
\end{array}\right),
$$

$d_{1}=2, d_{2}=4$. Take $p_{1}=p_{2}=q_{1}=q_{2}=0.5, \lambda=1.1, \eta_{1}=0.4, \eta_{2}=$ $0.4, \alpha_{1}=2.8, \alpha_{2}=1.4, \beta_{1}=1.5, \beta_{2}=1.8$, from the definition of $\tau$, we get that $\tau=0.6931$. It is easy to check that this example can not satisfy $\left(H_{1}\right)$. Meanwhile, This example can not satisfy Theorem 3.1, in addition, by Matlab software, we can know this system fails to maintain stable.

Remark 4.5 In this paper, we use the Matlab software to solve the examples, we can read the detailed methods reference from [34].

\section{Conclusions}

In this paper, we investigate mean-square exponential input-to-state stability of a class of stochastic recurrent neural networks with multi-proportional delays. Different from the prior works, delays here are proportional delays which are unbounded and time-varying. And the nonlinear activation functions are not necessarily differentiable, bounded, monotonic and nondecreasing, which the generalization for activation functions provides a wider application domain for neural networks. The nonlinear transformation $y(t)=x\left(\mathrm{e}^{t}\right)$ plays an important role, which will the considered system transform into stochastic recurrent neural networks with unequal constant delays and variable coefficient, so that 
the problem is simplified. This research method might have an impact role in studying dissipativity, periodicity and synchronization of recurrent neural networks with proportional delays.

\section{References}

[1] J. Liu, W. Luo, X. Yang, etc, Robust model-based fault diagnosis for PEM fuel cell air-feed system, IEEE Trans. Ind. Electron, 63(5) (2016) 3261-3270.

[2] J. Liu, S. Laghrouche, M. Wack, Observer-based higher order sliding mode control of power factor in three-phase $\mathrm{AC} / \mathrm{DC}$ converter for hybrid electric vehicle applications, Int. J. Control, 87(6) (2014) 1117-1130.

[3] J. Liu, S. Laghrouche, M. Harmouche, etc, Adaptive-gain second-order sliding mode observer design for switching power converters, Control Eng. Pract. 30(2014) 124-131.

[4] S. Young, P. Scott, N. Nasrabadi, Object recognition using multilayer Hopfield neural network, IEEE Trans. Image Process. 6(3)(1997) 357-372.

[5] D. Li, Q. Zhu, Comparison principle and stability of stochastic delayed neural networks with Markovian switching, Neurocomputing, 123 (2014) 436-442.

[6] E. Zhu, Q. Yuan, Pth moment exponential stability of stochastic recurrent neural networks with Markovian switching, Neural Process. Lett. $38(3)(2013) 487-500$.

[7] Y. Guo, H. Su, X. Ding, etc, Global stochastic stability analysis for stochastic neural networks with infinite delay and Markovian switching, Appl. Math. Comput. 245 (2014) 53-65.

[8] H. Zhang, Y. Wang, Stability analysis of Markovian jumping stochastic CohenGrossberg neural networks with mixed time delays, IEEE Trans. Neural Netw. 19(2)(2008) 366-370.

[9] R. Rakkiyappa, A. Chandrasekar, S. Lakshmanan, etc, Exponential stability of Markovian jumping stochastic Cohen-Grossberg neural networks with modedependent probabilistic time-varying delays and impulses, Neurocomputing, 131 (2014) 265-277.

[10] M. Hu, J. Cao, A. Hu, Mean square exponential stability for discretetime stochastic switched static neural networks with randomly occurring nonlinearities and stochastic delay, Neurocomputing, 19 (2014) 476-481.

[11] W. Chen, X. Lu, Mean square exponential stability of uncertain stochastic delayed neural networks, Physics Letters A 372(7) (2008) 1061-1069.

[12] G. Chen, O. Gaans, S.V. Lunel, Fixed points and Pth moment exponential stability of stochastic delayed recurrent neural networks with impulses, Appl. Math. Lett. 27 (2014) 36-42. 
[13] M.S. Ali, R. Saravanakumar, J. Cao, New passivity criteria for memristor-based neutral-type stochastic BAM neural networks with mixed time-varying delays, Neurocomputing, 171 (2015) 1533-1547.

[14] R. Saravanakumar, M.S. Ali, M. Hua, $H_{\infty}$ state estimation of stochastic neural networks with mixed time-varying delays, Soft Computing, (2015) DOI: 10.1007/s00500-015-1901-4.

[15] G. Peng, L. Huang, Exponential stability of hybrid stochastic recurrent neural networks with time-varying delays, Nonlinear Anal. 2(4) (2008) 1198-1204.

[16] G. Sun, Y. Zhang, Exponential stability of impulsive discrete-time stochastic BAM neural networks with time-varying delay, Neurocomputing, 131 (2014) 323-330.

[17] X. Meng, M. Tian, S. Hu, Stability analysis of stochastic recurrent neural networks with unbounded time-varying delays, Neurocomputing, 74(6) (2011) 949-953.

[18] W. Zhang, C. Li, T. Huang, J. Qi, Global exponential synchronization for coupled switched delayed recurrent neural networks with stochastic perturbation and impulsive effects, Neural Comput. Appl. 25(6) (2014) 12751283.

[19] L. Sheng, M. Gao, H. Yang, Delay-dependent robust stability for uncertain stochastic fuzzy Hopfield neural networks with time-varying delays, Fuzzy Set. Syst. 160(24)(2009) 3503-3517.

[20] S. Zhu, Y. Shen, Two algebraic criteria for input-to-state stability of recurrent neural networks with time-varying delays, Neural Comput. Appl. 22(6) (2013) 1163-1169.

[21] X. Su, P. Shi, L. Wu, etc, Reliable filtering with strict dissipativity for T-S fuzzy time-delay systems, IEEE Trans. Cyb. 44(12)(2014) 2470-2483.

[22] Z. Yang, W. Zhou, T. Huang, Exponential input-to-state stability of recurrent neural networks with multiple time-varying delays, Cogn. Neurodynamic, $8(1)(2014) 47-54$.

[23] Q. Zhu, J. Cao, Mean-square exponential input-to-state stability of stochastic delayed neural networks, Neurocomputing, 131 (2014) 157-163.

[24] O. Naifar, A.B. Makhlouf, M.A. Hammami, etc, State feedback control law for a class of nonlinear time-varying system under unknown time-varying delay, Nonlinear Dyn. 82(1) (2015) 349-355.

[25] J.A. Melo, J.M. Adán-Coello, WBWTP+: A packet scheduling algorithm for achieving proportional delay differentiation in IP networks, Salesian J. Inform. Syst. 11 (2013) 27-32.

[26] B. Izhak, H. Ortal, Optimal phase calibration of nonlinear delayed sensors, Mech. Syst. Signal Process. 45(2) (2014) 424-432. 
[27] L. Fox, D.F. Mayers, On a functional differential equational, J. Appl. Math. 8(3) (1971) 271-307.

[28] Y.K. Liu, Asymptotic behavior of functionoal differential equations with proportional time delays, European J. Appl. Math. 7(1) (1994) 11-30.

[29] L. Zhou, Dissipativity of a class of cellular neural networks with proportional delays, Nonlinear Dyn. 2013;73(3):1895-1903.

[30] L. Zhou, Delay-dependent exponential stability of cellular neural networks with multi-proportional delays, Neural Process. Lett. 38(3) (2013) 321-346.

[31] L. Zhou, Global asymptotic stability of cellular neural networks with proportional delays, Nonlinear Dyn.77(1)(2014) 41-47.

[32] L. Zhou, X. Chen, Y. Yang, Asymptotic stability of cellular neural networks with multi-proportional delays, Appl. Math. Comput. 229(1) (2014) 457-46.

[33] X. Song, J. Peng, Global asymptotic stability of impulsive CNNs with proportional delays and partially Lipschitz activation functions, Abstract Appl. Anal. 2014 (2014) DOI:10.1155/2014/83.

[34] L. Zhou, Novel global exponential stability criteria for hybrid BAM neural networks with proportional delays, Neurocomputing. 229(15) (2015) 99-106.

[35] L. Zhou, Y. Zhang, Global exponential periodicity and stability of recurrent neural networks with multi-proportional delays, ISA Trans. 60(1)(2016) 89-95.

[36] L. Zhou, Y. Zhang, Global exponential stability of a class of impulsive recurrent neural networks with proportional delays via fixed point theory, J. Franklin Institute 353(2) (2016) 561-575. 